\title{
Effects of miR-195-5p on cell proliferation and apoptosis in gestational diabetes mellitus via targeting EZH2
}

\author{
XIAOJIE LIAO $^{1}$, ZHUOLIN ZHOU $^{2}$ and XIAOLIU ZHANG ${ }^{1}$ \\ ${ }^{1}$ Department of Obstetrics, Jingmen No. 1 People's Hospital, Jingmen, Hubei 448000; ${ }^{2}$ Family Planning Ward, \\ The First Affiliated Hospital of Guangxi Medical University, Nanning, Guangxi 530000, P.R. China
}

Received September 26, 2019; Accepted February 6, 2020

DOI: $10.3892 / \mathrm{mmr} .2020 .11142$

\begin{abstract}
Gestational diabetes mellitus (GDM) is a type of diabetes mellitus (DM) that occurs during pregnancy. The present study aimed to investigate the roles of microRNA (miR)-195-5p and enhancer of zeste homolog 2 (EZH2) in GDM, and their potential association. Human umbilical vein endothelial cells (HUVECs) were collected from healthy and GDM umbilical cords, and the endothelial properties were detected by flow cytometry. mRNA expression levels of miR-195-5p and EZH2, and EZH2 protein expression levels were detected by reverse transcription-quantitative PCR (RT-qPCR) and western blot analysis, respectively. Cell colony formation and flow cytometry were performed to determine cell proliferation and apoptosis. Furthermore, the target gene of miR-195-5p was predicted and assessed using a dual-luciferase reporter assay. The levels of cell viability, proliferation and apoptosis following the overexpression of miR-195-5p, EZH2 or miR-195-5p + EZH2, were detected using Cell Counting Kit-8, colony formation and flow cytometry assays, respectively. In addition, the mRNA expression levels of miR-195-59 and $\mathrm{EZH} 2$, and $\mathrm{EZH} 2$ protein expression levels following transfection with overexpression plasmids were detected using RT-qPCR and western blot analysis, respectively. It was identified that high mRNA expression of miR-195-5p, and low EZH2 mRNA and protein expression levels decreased the level of cell proliferation and the high apoptotic rate of GDM-HUVECs. In addition, miR-195-5p was predicted and identified to target EZH2, and miR-195-5p overexpression was identified to inhibit cell proliferation and promote apoptosis.
\end{abstract}

Correspondence to: Dr Xiaoliu Zhang, Department of Obstetrics, Jingmen No. 1 People's Hospital, 168 Xiangshan Avenue, Jingmen, Hubei 448000, P.R. China

E-mail: xiaoliuz_zhangx1@163.com

Abbreviations: GDM, gestational diabetes mellitus; DM, diabetes mellitus; HUVECs, human umbilical vein endothelial cells; miRNAs, microRNAs

Key words: microRNA-195-5p, enhancer of zeste homolog 2, cell proliferation, apoptosis, gestational diabetes mellitus
However, it was demonstrated that upregulation of EZH2 could alleviate the inhibition of cell proliferation and the increased apoptotic rate induced by miR-195-5p overexpression. Therefore, the present results suggested that miR-195-5p may inhibit cell viability, proliferation and promote apoptosis by targeting EZH2 in GDM-induced HUVECs.

\section{Introduction}

Gestational diabetes mellitus (GDM) is a type of diabetes mellitus (DM) that develops in pregnant women as the pregnancy progresses, and can be caused by maternal hyperglycemia and decreased glucose intolerance (1). A high glucose concentration in the internal environment caused by maternal hyperglycemia is dangerous to the fetus, as glucose can be easily transferred via the fetus-placenta circulation. However, maternal insulin cannot be transferred across the placental barrier, and therefore under high glucose conditions the fetus experiences fetal hyperinsulinemia, leading to fetal disorders (2). Previous studies have demonstrated that despite recovery following pregnancy, mothers with GDM are more likely to have type $2 \mathrm{DM}$ and other cardiovascular diseases (3). In addition, phenotypic changes induced by GDM can persist in infants carried by mothers with GDM, and these infants are more likely to have DM or obesity in later life (4). Therefore, epigenetic modifications may be involved in the GDM-induced high glucose environment and further accentuating the physical consequence of GDM (5).

MicroRNAs (miRNAs) are small non-coding RNAs that can modulate gene expressions at the post-transcriptional level. miRNAs (miR) have been widely studied in tumor research, as they are closely associated with tumorigenesis and the development of numerous cancer types. In cervical cancer, miR-125a serves as a tumor suppressor by inhibiting tumor growth, invasive ability and metastasis via sponging STAT3 (6). Furthermore, miR-193a inhibits cell proliferation and metastasis in breast cancer by targeting WT1 transcription factor (7). However, to the best of our knowledge, there are few studies investigating the association between miRNAs and GDM. In total, 6 miRNAs: miR-155-5p; -21-3p; -146b-5p; $-223-3 p$; $-517-5 p$; and $\mathrm{miR}-29 \mathrm{a}-3 \mathrm{p}$, may have the potential to increase the likelihood of GDM occurrence in pregnant women (8). In addition, a miRNA profiling analysis in patients with GDM revealed that miR-195-5p exhibited the highest fold 
upregulation in GDM and was involved in metabolism (9). However, the function and mechanism of miR-195-5p in human umbilical vein endothelial cells (HUVECs) with GDM remains unknown.

It has been hypothesized that epigenetic changes induce gene expression deregulation in response to perturbations of the utero environmental (10). Enhancer of zeste homolog 2 (EZH2) is a component of the polycomb repressor complex 2 (PRC2). Moreover, PRC2 is an epigenetic regulator that initiates and maintains the trimethylation of histone $\mathrm{H} 3$ on lysine 27 (H3K27me3), which is an epigenetic marker associated with heterochromatin formation and transcription silencing $(11,12)$. A previous study demonstrated that EZH2 is a target of GDM and mediates the damaging effects of GDM on HUVECs (10). However, the function of EZH2 on cell survival of GDM-HUVECs and its association with miR-195-5p are not fully understood.

Therefore, the aim of the present study was to investigate the effects of miR-195-5p and EZH2, a hypothesized target gene for miR-195-5p, on HUVECs.

\section{Materials and methods}

Patient recruitment and ethical approval. Human umbilical cords were obtained from 25 full-term healthy women (age, 35-23) and 25 women with GDM (age, 45-25) in Jingmen No. 1 People's Hospital from February 2017 to March 2018. The diagnosis of GDM was based on the 2010 GDM diagnosis criteria introduced by International Association of The Diabetes and Pregnancy Study Group (13). The study was approved by Jingmen No. 1 People's Hospital Ethics Committee (approval no., NJ201702463) and was performed in accordance with The Declaration of Helsinki (1964) and its later amendments. All patients provided written informed consent.

HUVECs. HUVECs derived from umbilical cords were prepared (14). Umbilical cord veins were rinsed in PBS, and HUVECs were incubated with $0.05 \%$ collagenase type II derived from Clostridium histolyticum; (Sigma-Aldrich; Merck KGaA) in M199 medium (Invitrogen; Thermo Fisher Scientific Inc.) containing $100 \mathrm{U} / \mathrm{ml}$ penicillin $\mathrm{G}$ sodium salt and $100 \mu \mathrm{g} / \mathrm{ml}$ streptomycin sulfate (Sigma-Aldrich; Merck $\mathrm{KGaA}$ ) at $37^{\circ} \mathrm{C}$ with $5 \% \mathrm{CO}_{2}$ for $10 \mathrm{~min}$. Cells were centrifugated at $1,000 \mathrm{x} \mathrm{g}$ for $10 \mathrm{~min}$ at room temperature, and then resuspended in M199 medium containing 10\% FBS (Invitrogen; Thermo Fisher Scientific, Inc.) and 10\% newborn calf serum (Invitrogen; Thermo Fisher Scientific, Inc.), $2 \mathrm{mM}$ glutamine and antibiotics as mentioned above at $37^{\circ} \mathrm{C}$ with $5 \% \mathrm{CO}_{2}$. For detecting cell endothelial properties, cells were cultured in endothelial cell basal medium (Lonza Group AG) containing $10 \% \mathrm{FBS}$ at $37^{\circ} \mathrm{C}$ for $24 \mathrm{~h}$ and a EGM-2 Epithelial SingleQuots ${ }^{\mathrm{TM}}$ kit (cat. no. CC-4176; Lonza Group AG) was used. The cells were fixed with $1 \%$ paraformaldehyde at $37^{\circ} \mathrm{C}$ for $30 \mathrm{~min}$. The cells were then analyzed using a flow cytometer (FACS Canto II; BD Biosciences) with cellQuest software (version 7.5.3; BD Biosciences), with mouse anti-human phycoerythrin-platelet endothelial cell adhesion molecule (CD31) antibody (cat. no. MHCD3104; eBioscience; Thermo Fisher Scientific, Inc.; 1:100) at $4^{\circ} \mathrm{C}$ for 20 min used as an endothelial cell marker.
Cell Counting Kit-8 assay (CCK-8). After $24 \mathrm{~h}$ cell cultivation, a cell solution with a density of $3 \times 10^{4}$ cells $/ \mathrm{ml}$ was prepared and cultured in 96 -well plates at $37^{\circ} \mathrm{C}$ for $24 \mathrm{~h}$. Then, cell viability was determined using a CCK- 8 assay (Dojindo Molecular Technologies, Inc.) according to the manufacturer's protocol, and optical density value was measured at the wavelength of $450 \mathrm{~nm}$.

Colony formation assay. HUVECs were lysed using $0.25 \%$ trypsin-0.02\% EDTA (Gibco; Thermo Fisher Scientific, Inc.), and the cell suspension was prepared and seeded $\left(1 \times 10^{4}\right)$ into 6-well plate for 10 days at $37^{\circ} \mathrm{C}$. HUVECs were washed with PBS 3 times, and cells were fixed with $10 \%$ methanol under room temperature for $15 \mathrm{~min}$ and stained with $0.1 \%$ crystal violet solution at room temperature for $30 \mathrm{~min}$. Images of the cells were captured (light microscope; magnification, x1), The number of colonies were counted using a light microscope (Olympus CKX41; Olympus Corporation).

Flow cytometry assay. Cell suspension was prepared in $500 \mu \mathrm{l}$ binding buffer (Gibco; Thermo Fisher Scientific, Inc.). Cell staining was performed with Annexin V-fluorescein isothiocyanate and propidium iodide $(20 \mu \mathrm{g} / \mathrm{ml}$; Biovision, Inc.) to determine the apoptotic rate of HUVECs in the dark for $15 \mathrm{~min}$ at room temperature. Cells were analyzed by flow cytometry (FACSCalibur; BD Biosciences) with BD CellQuest Pro Software version 1.2 (BD Biosciences) in the dark.

Cell transfection. pCMV6-XL5-EZH2 (cat. no. SC101257) and its empty control vector were purchased from OriGene Technologies, Inc. miR-195-5p mimics (cat. no. miR10000461-1-5, 5'-CGGUUAUAA AGACACGAC GAU-3') and its negative control RNA were obtained from Guangzhou RiboBio Co., Ltd. HUVECs were incubated to $70-80 \%$ confluence at $37^{\circ} \mathrm{C}$ for $24 \mathrm{~h}$. A total of $20 \mu \mathrm{M}$ miR-195-5p mimics, negative control (NC), miR-195-5p mimic, mimic + EZH2 or EZH2 were used and the transfection procedure was conducted using Lipofectamine 2000 (Invitrogen; Thermo Fisher Scientific, Inc.). After 24 h, the cells were used to perform the subsequent experiments.

Dual-luciferase reporter assay. The 3'-untranslated regions (UTR) of EZH2 contained a predicted binding-site for miR-195-5p, as identified using Targetscan7.2 (http://www.targetscan.org/vert_72/) analysis. To assess whether miR-195-5p specifically targets EZH2, this was examined using the luciferase pGL3-Basic vector (Promega Corporation). Firstly, wild-type (WT) and mutant (MUT) EZH2-3'-UTR were purchased from Shanghai GenePharma Co., Ltd. and inserted into the luciferase vector. Then, the miR-195-5p mimic was co-transfected with WT or MUT luciferase vector into $293 \mathrm{~T}$ cells (American Type Culture Collection) using Lipofectamine ${ }^{\circledR} 2000$ (Invitrogen; Thermo Fisher Scientific, Inc.). After $48 \mathrm{~h}$ of transfection, luciferase activity was measured by the Dual-Luciferase Reporter Assay System (Promega Corporation). Renilla luciferase activity was detected in the same method.

Reverse transcription-quantitative polymerase chain reaction $(R T-q P C R)$. miR-195-5p and EZH2 mRNA expression levels in 
HUVECs were determined by RT-qPCR. Total RNA of EZH2 was harvested using QIAzol Lysis reagent (Qiagen, Inc.), and total RNAs of miR-195-5p was isolated using miRVana miRNA Isolation kit (Thermo Fisher Scientific, Inc.). RT of EZH2 and miR-195-5p from RNAs to cDNAs was performed using a QuantiTect RT kit (Qiagen, Inc.) and TaqMan miRNA RT kit (Thermo Fisher Scientific, Inc.) at $37^{\circ} \mathrm{C}$ for $45 \mathrm{~min}$ and then at $80^{\circ} \mathrm{C}$ for $5 \mathrm{~min}$. The Step One Plus RT PCR system (Applied Biosystems; Thermo Fisher Scientific, Inc.) was used to perform RT-qPCR. The PCR reaction was as follows: Initital denaturation at $95^{\circ} \mathrm{C}$ for $5 \mathrm{~min}$, followed by 40 cycles at $94^{\circ} \mathrm{C}$ for $30 \mathrm{sec}, 55^{\circ} \mathrm{C}$ for $30 \mathrm{sec}, 72^{\circ} \mathrm{C}$ for $45 \mathrm{sec}$ and $72^{\circ} \mathrm{C}$ extension for $10 \mathrm{~min}$. Data were analyzed using $2^{-\Delta \Delta \mathrm{Cq}}$ method (15). GAPDH and U6 served as internal references for EZH2 and miR-195-5p, respectively. The primers sequences used are shown in Table I.

Western blot analysis. Following rinsing the cells in cold PBS 3 times, HUVECs were lysed in lysis buffer containing $10 \mathrm{mM}$ HEPES (pH 7.5), $10 \mathrm{mM} \mathrm{KCl}, 1.5 \mathrm{mM} \mathrm{MgCl}_{2}, 0.34 \mathrm{M}$ sucrose, $10 \%$ glycerol and $0.1 \%$ TritonX-100 with protease inhibitors at $4^{\circ} \mathrm{C}$. Total proteins were extracted and the concentration was determined with a bicinchoninic acid assay kit (Thermo Fisher Scientific, Inc.). Proteins ( $20 \mu \mathrm{g} / \mathrm{lane})$ were isolated on $10 \%$ SDS-PAGE and then transferred into PVDF membranes, which were blocked with 5\% skimmed milk powder at room temperature for $2 \mathrm{~h}$. Blots were incubated overnight at $4^{\circ} \mathrm{C}$ with the primary antibody against EZH2 (cat. no. GTX110384; 1:500; GeneTex, Inc.) and GAPDH (cat. no. GTX100118; 1:5,000; GeneTex, Inc.). Following incubation, the membranes were washed three times with TBST $(0.05 \%$ Tween 20$)$ and cultured with horseradish peroxidase-conjugated goat anti-rabbit immunoglobulin G (cat. no., GTX213110-01; 1:1,000; GeneTex, Inc.) for $2 \mathrm{~h}$ at room temperature. The bands were detected by chemiluminescence (ECL ${ }^{\mathrm{TM}}$ Prime; GE Healthcare Life Sciences), imaged on X-ray film (GE Healthcare Lifesciences) and quantified using ImageJ (version 1.8.0; National Institutes of Health).

Statistical analysis. All experiments were repeated in triplicate. Statistical analyses were performed using SPSS v.19.0 software (IBM Corp.), and GraphPad Prism v.5.02 software (GraphPad Prism Software, Inc.) was used to create the graphs. Data are presented as the mean \pm standard deviation, and were evaluated using unpaired Student's t-test in luciferase activity assay, independent samples t-test in comparison with Healthy and GDM groups or analysis of variance followed by Tukey's post-hoc test. $\mathrm{P}<0.05$ was considered to indicate a statistically significant difference.

\section{Results}

GDM-induced phenotypic alterations in HUVECs. The endothelial phenotype of HUVECs indicated by CD31 was detected by flow cytometry, and $>95 \%$ of GDM-HUVECs and healthy HUVECs were identified as CD31-positive (Fig. 1A). Moreover, compared with the healthy controls, it was identified that miR-195-5p mRNA expression in GDM-HUVECs was significantly increased, but EZH2 mRNA and protein expression levels were significantly decreased (Fig. 1B-E).
Table I. Primers sequence used for reverse transcriptionquantitative PCR.

\begin{tabular}{ll}
\hline Primer & \multicolumn{1}{c}{ Sequence } \\
\hline EZH2 & \\
Forward & 5'-CCTGAAGTATGTCGGCATCGAAAGAG-3' \\
Reverse & 5'-TGCAAAAATTCACTGGTACAAAACACT-3' \\
miR-195-5p & \\
Forward & 5'-GTCGTATCCAGTGCAGGGTCCGAGGT-3' \\
Reverse & 5'-ATTCGCACTGGATACGACTATAACCG-3' \\
U6 & \\
Forward & 5'-CTCGCTTCGGCAGCACA-3' \\
Reverse & 5'-AACGCTTCACGAATTTGCGT-3' \\
GAPDH & \\
Forward & 5'-CGGAGTCAACGGATTTGGTCGTAT-3' \\
Reverse & 5'-AGCCTTCTCCATGGTGGTGAAGAC-3' \\
\hline
\end{tabular}

miR, microRNA; EZH2, enhancer of zeste homolog 2.

In addition, decreased cell proliferation (Fig. 1F and G) and elevated apoptosis were identified in GDM-HUVECs (Fig. $1 \mathrm{H}$ and I).

miR-195-5p targets EZH2. EZH2 was predicted to be the target gene of miR-195-5p (Fig. 2A), which was confirmed using a dual-luciferase reporter assay. Furthermore, it was identified that cells transfected with miR-195-5p mimics exhibited significantly decreased luciferase activity compared with the blank group in the EZH2-WT group (Fig. 2B).

Effects of miR-195-5p and EZH2 on functional capacities of control HUVECs. Cell viability, proliferation and apoptosis were detected following overexpression of miR-195-5p, EZH2 or the combination of both in control HUVECs (Fig. 3A-E). It was demonstrated that miR-195-5p overexpression suppressed cell viability and proliferation, but increased the apoptotic rate. However, overexpression of EZH2 promoted cell viability and proliferation, and inhibited apoptosis. In addition, the combination of the overexpression of miR-195-5p and EZH2 was identified to partially alleviate the effects caused by miR-195-5p overexpression on cell viability, proliferation and apoptosis.

Effects of miR-195-5p and EZH2 on the expression levels of miR-195-5p and EZH2 in control HUVECs. The present results suggested that the expression of miR-195-5p was upregulated by miR-195-5p overexpression, while EZH2 overexpression had no significant effect on miR-195-5p (Fig. 3F). Furthermore, EZH2 expression was affected by overexpression of both miR-195-5p and EZH2, as upregulation of EZH2 promoted EZH2 expression, while miR-195-5p overexpression downregulated EZH2 (Fig. 3G-I).

\section{Discussion}

In pregnant women with DM, exposure to hyperglycemia will result in long-lasting effects to the vascular cells, which 
A

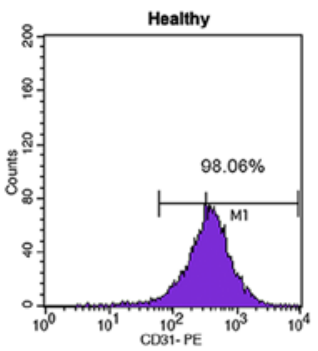

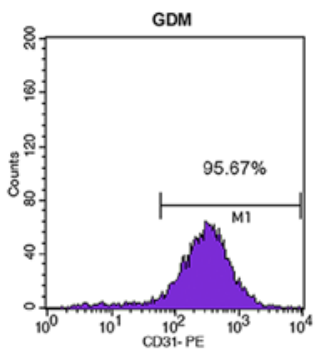

B

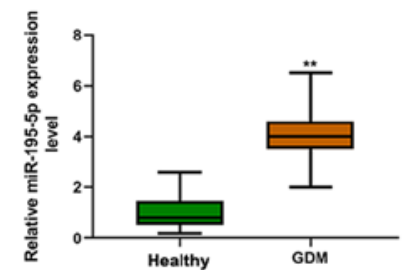

C

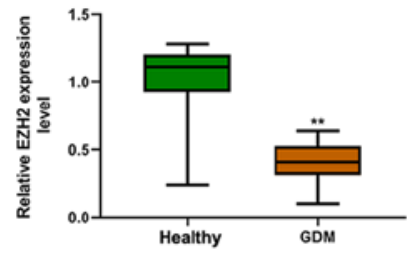

D

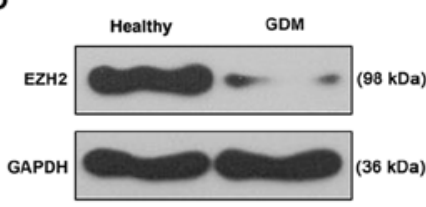

$E$

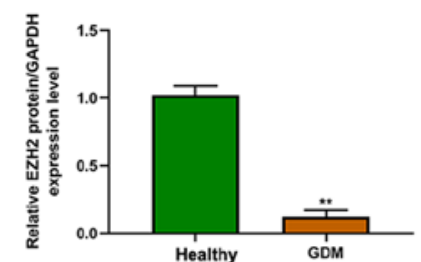

$\mathbf{F}$

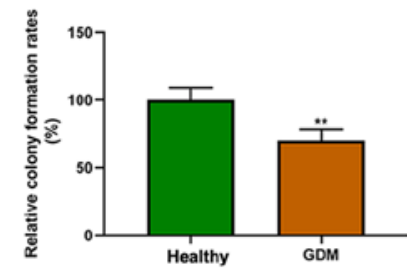

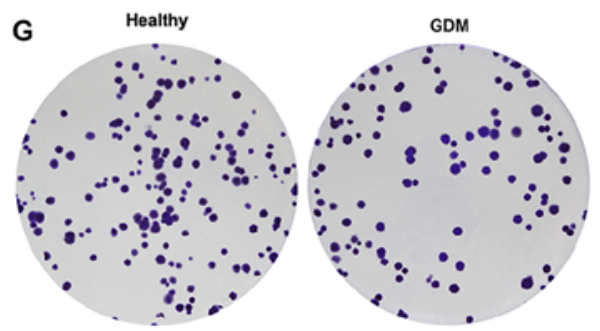
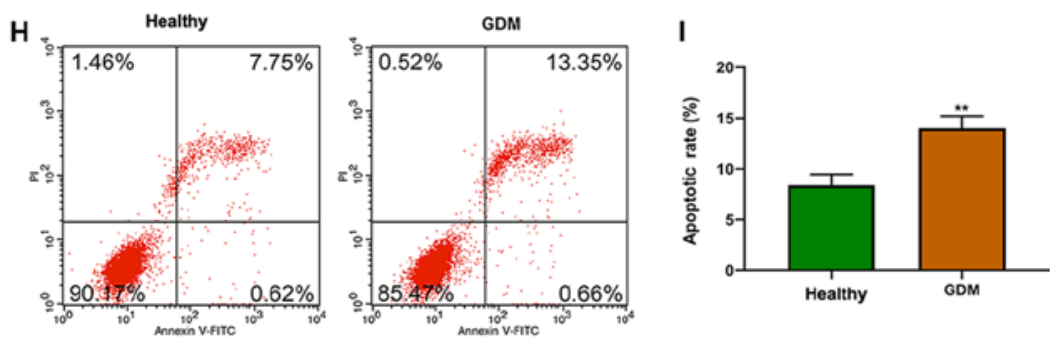

Figure 1. Expression levels of miR-195-5p and EZH2, and cell proliferation and apoptosis in healthy controls and GDM HUVECs. (A) Endothelial phenotype of healthy controls and GDM HUVECs was detected by flow cytometry using the CD31 marker. mRNA expression levels of (B) miR-195-5p and (C) EZH2 were determined by reverse transcription-quantitative polymerase chain reaction analysis. (D) Protein expression of EZH2 determined by western blot analysis. (E) Quantification of the western blot analysis densitometric data. (F) Cell proliferation was determined by colony formation assay. (G) Representative images of cell colonies. (H) Flow cytometry was used to assess cell apoptosis. (I) Quantification of the flow cytometry data. ${ }^{* *} \mathrm{P}<0.01$ vs. Healthy group. miR, microRNA; EZH2, enhancer of zeste homolog 2; Gestational diabetes mellitus; HUVECs, human umbilical vein endothelial cells; CD31, platelet endothelial cell adhesion molecule; PE, phycoerythrin.

A

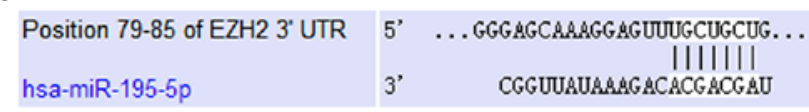

B

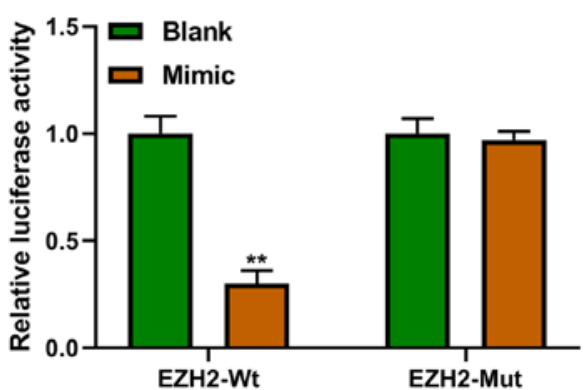

Figure 2. Prediction and assessment of miR-195-5p target gene. (A) Prediction of miR-195-5p target gene. (B) Assessment of miR-195-5p target gene using a dual-luciferase reporter assay. ${ }^{* *} \mathrm{P}<0.01$ vs. Blank. miR, microRNA; EZH2, enhancer of zeste homolog 2; WT, wild-type; Mut, mutant; 3'UTR, 3'untranslated region; hsa, Homo sapiens.

can cause vascular complications $(16,17)$. The underlying epigenetic mechanisms, involving DNA modification and histone marks, affect the activation of gene transcription (10). These epigenetic modifications enable the cells to respond to changing internal and external environments and adjust to these environmental stimulations. Initially, epigenetics was referred to as to chromatin changes that were inherited; however, this definition has been expanded, and epigenetics are now known to have long-term effects, from pregnancy through to every stage of human growth (18). In addition, epigenetic modification can be altered (strengthened, alleviated or even erased) over time due to the effects of drug use, change in lifestyles or other environment stimuli (10). DM is closely associated with the genome (19), and miR-216 may be a potential therapeutic agent for DM due to its role in angiogenesis and its protective effects on vascular integrity in preventing the pathogenesis and complications of DM (20). Furthermore, miR-155, miR-146a and long non-coding RNA homeodomain interacting protein kinase 3 have been demonstrated to serve important roles in the dysfunction of type 1 or type 2 DM (21-23). Unlike DM, the changes to glucose metabolism caused by GDM during pregnancy can be reverted following childbirth (24). However, GDM is associated with increased cardiovascular risk and type $2 \mathrm{DM}$ in both the mothers and their children in later life (24).

Endothelial dysfunction is a common feature in numerous pregnancy-associated diseases, as well as in the different forms of DM (25). HUVECs from human umbilical cords are widely used as a cellular model for the analysis of the effects of uterine environmental changes on fetal endothelium. Previous GDM data have demonstrated that trophoblasts from human term placenta exhibit high apoptotic rates, and that maternal 
A

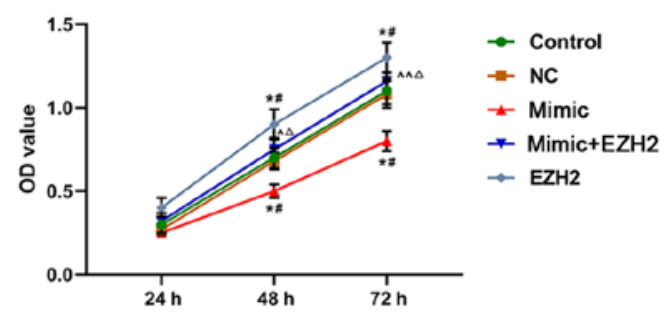

B

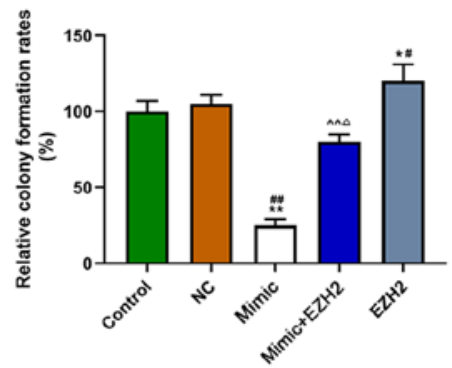

C

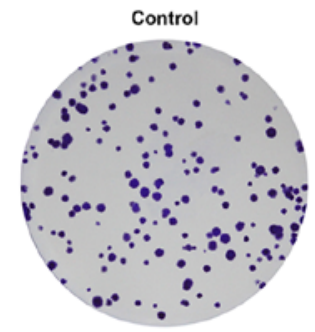

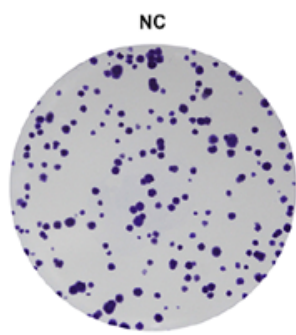
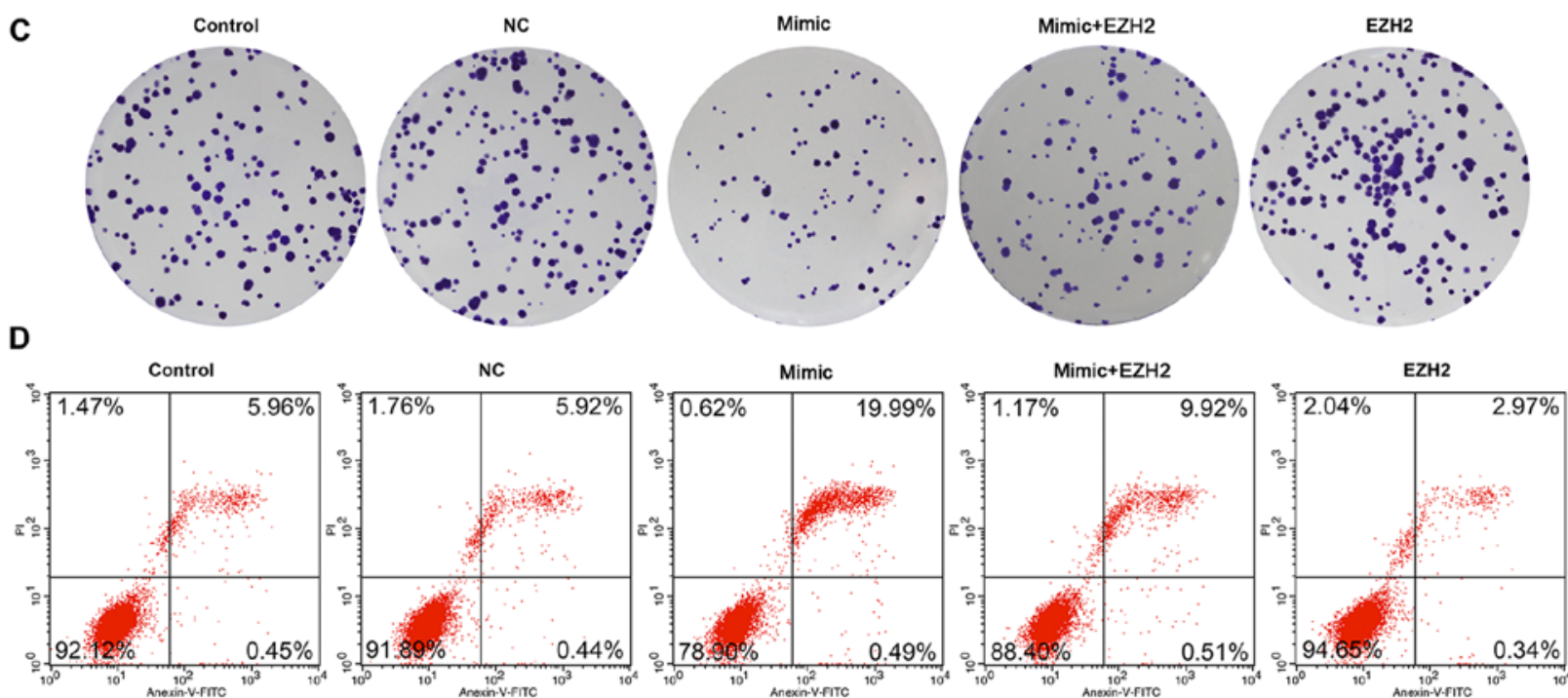

E

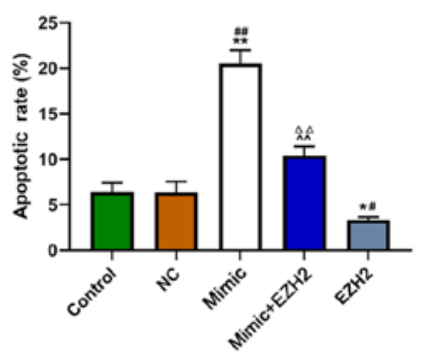

$\mathrm{F}$

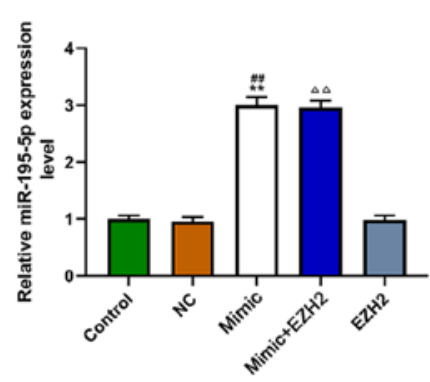

6

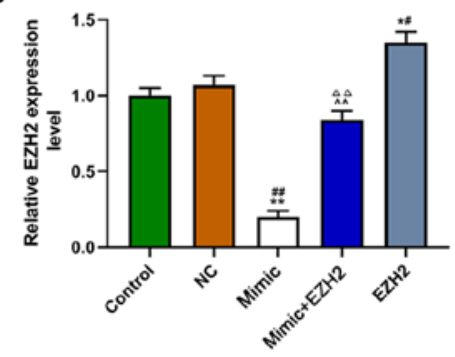

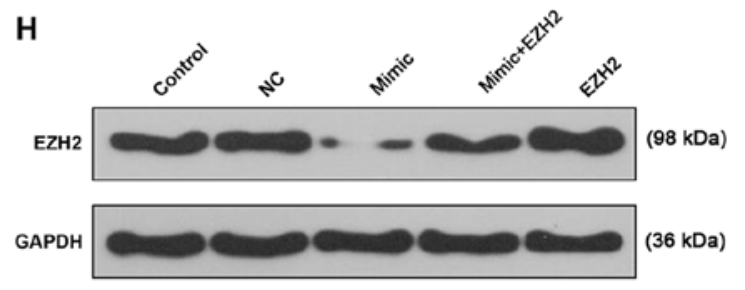

I

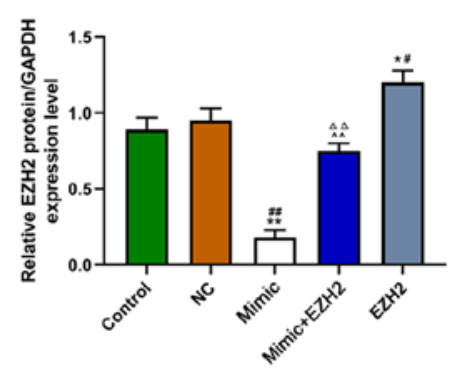

Figure 3. Effects of miR-195-5p and EZH2 on cell viability, proliferation and apoptosis in healthy HUVECs. Groups were divided into control (cells treated with PBS), NC, miR-195-5p mimic, mimic + EZH2 and EZH2 groups. (A) Cell viability in HUVECs determined using a Cell Counting Kit-8 assay. (B) Cell proliferation in HUVECs determined by colony formation assays. (C) Representative images of cell colonies (D) Flow cytometry was used to assess cell apoptosis in HUVECs. (E) Quantification of the flow cytometry data. (F) miR-195-5p mRNA expression in HUVECs was determined by RT-qPCR. (G) RT-qPCR and $(\mathrm{H})$ western blot analysis were used to determine EZH2 mRNA and protein expression levels in HUVECs. (I) Quantification of the western blot analysis densitometric data. ${ }^{*} \mathrm{P}<0.05,{ }^{* *} \mathrm{P}<0.01$ vs. Control. ${ }^{\|} \mathrm{P}<0.05,{ }^{\# \#} \mathrm{P}<0.01$ vs. $\mathrm{NC} .{ }^{\wedge} \mathrm{P}<0.05,{ }^{\wedge} \mathrm{P}<0.01$ vs. Mimic; ${ }^{\Delta} \mathrm{P}<0.05,{ }^{\Delta \Delta} \mathrm{P}<0.01$ vs. EZH2. miR, microRNA; EZH2, enhancer of zeste homolog 2; GDM, gestational diabetes mellitus; NC, negative control; HUVECs, human umbilical vein endothelial cells; RT-qPCR, reverse transcription-quantitative polymerase chain reaction; FITC, fluorescein isothiocyanate; PI, propidium iodide; OD, optical density.

GDM is associated with alternations in apoptotic and inflammatory gene expression levels (26). Furthermore, proliferation of pancreatic $\beta$ cells is inhibited by decreases in placental growth factor in GDM (27).
The association between GDM and miRNAs has also been previously investigated: miRNA expression levels in HUVECs were associated with the occurrence of GDM, and alternations of miRNAs in HUVECs are speculated to affect 
metabolic processes of GDM (28). A previous study revealed that miR-195-5p was significantly upregulated in patients with deep vein thrombosis, which is one of the most common cardiovascular diseases (29). In addition, it has been demonstrated that the expression of miR-195-5p in GDM is significantly increased in comparison with the healthy group (9). Similar to these data concerning deep vein thrombosis, the results of the present study identified that miR-195-5p was also significantly upregulated in GDM. In addition, the downstream target gene of miR-195-5p was identified to be EZH2, which elicited the opposite effects on cellular processes compared with miR-195-5p: It was identified that high expression of miR-195-5p decreased cell viability and proliferation, and promoted apoptosis, while EZH2 overexpression resulted in the opposite effects. Furthermore, the combination of both factors partially attenuated the previous inhibition of cell growth and promotion of cell apoptosis induced by the upregulation of miR-195-5p.

Previous studies have indicated that EZH2 enhances the proliferative capacity of gastric cancer cells by suppressing cyclin-dependent kinase inhibitor 1 (p21) expression $(30,31)$. Furthermore, in laryngeal carcinoma, EZH2 promotes cell proliferation by regulating Runt-related transcription factor 3 (RUNX3) (32). It has also been demonstrated that EZH2 promotes the epigenetic silencing of miR-205 and miR-31 to suppresses apoptosis (1). Therefore, EZH2 can regulate proliferation and apoptosis in multiple cell types. In concordance with these previous studies, the results of the present study indicated that EZH2 was downregulated in GDM, and that overexpression of EZH2 enhanced proliferation and inhibited apoptosis of HUVECs. Moreover, we hypothesized that EZH2 may regulate proliferation and apoptosis by directly modulating the cell cycle, proliferation, and apoptotic-associated genes or miRNAs, including p21, RUNX3, miR-205 and miR-31.

Previous studies have focused on the role of miR-195-5p in tumorigenesis: In hepatocellular carcinoma, miR-195 can act as a tumor suppressor via the inhibition of cell proliferation by targeting astrocyte elevated gene-1 (33). In papillary thyroid carcinoma, it was demonstrated that miR-195 inhibited tumor growth and prevented metastasis via sponging cyclin D1 and fibroblast growth factor 2, suppressing tumor development (34). Furthermore, EZH2 was identified to be closely associated with cancer, with regards to its therapeutic effects on cell proliferation, invasion and metastasis (35). Moreover, by suppressing the expression of EZH2, which promotes the phosphorylation process in breast cancer cells, the progression of breast cancer is markedly inhibited (36). In GDM-HUVECs, EZH2 can affect the regulation of $\beta$-cells via the interaction with prolactin receptor genes (37). A previous study has also revealed that EZH2 is downregulated in GDM-HUVECs, and that the overexpression of EZH2 inhibits apoptosis and promotes migration by targeting the miR-101 promoter for inhibition of HUVECs (10). Therefore, based on previous data and the results from the present study, we hypothesized that miR-195-5p and EZH2 may participate in cellular processes of HUVECs, and the interaction of these 2 factors affects the progression of GDM. EZH2 was demonstrated to suppress the expression of miR-195-5p in cervical cancer cells via increasing H3K27me3 in the promoter region of miR-195 (31), which is different from the present results. Therefore, it is hypothesized that the association between miR-195-5p and EZH2 may be complicated, and vary between different cell types. Therefore, further studies are required to investigate the mechanism underlying the association between miR-195-5p and EZH2. Furthermore, additional indicators associated with GDM-HUVECs should be assessed in future experiments, such as changes in apoptotic and inflammatory gene expression levels, metabolic product levels and angiogenesis. Moreover, the function of miR-195-5p and EZH2 in GDM should be further examined in an animal model.

In conclusion, the present study investigated the role of miR-195-5p in HUVECs with GDM, and identified that miR-195-5p may be associated with cell proliferation and apoptosis of HUVECs by sponging EZH2. The present results provide evidence of a novel underlying mechanism involving miRNA and epigenetic regulation in GDM, which may facilitate the development of potential therapeutic strategies for GDM.

\section{Acknowledgements}

Not applicable.

\section{Funding}

No funding was received.

\section{Availability of data and materials}

The datasets used and/or analyzed during the current study are available from the corresponding author on reasonable request.

\section{Authors' contributions}

XL made substantial contributions to conception and design of the project. ZZ, XZ and XL were responsible for data acquisition, data analysis and interpretation. XL was involved in drafting the article and critically revising it for important intellectual content. All authors read and approved the final version of the manuscript. All authors agreed to be accountable for all aspects of the work in ensuring that questions related to the accuracy or integrity of the work are appropriately investigated and resolved.

\section{Ethics approval and consent to participate}

The present study was approved by Jingmen No. 1 People's Hospital Ethics Committee (approval no. NJ201702463) and was performed in accordance with The Declaration of Helsinki (1964) and its later amendments. All patients provided written informed consent.

\section{Patient consent for publication}

All patients provided written informed consent.

\section{Competing interests}

The authors declare that they have no competing interests. 


\section{References}

1. Zhang Q, Padi SK, Tindall DJ and Guo B: Polycomb protein EZH2 suppresses apoptosis by silencing the proapoptotic miR-31. Cell Death Dis 5: e1486, 2014.

2. Desoye G and Nolan CJ: The fetal glucose steal: An underappreciated phenomenon in diabetic pregnancy. Diabetologia 59: 1089-1094, 2016.

3. Leach L: Placental vascular dysfunction in diabetic pregnancies: Intimations of fetal cardiovascular disease? Microcirculation 18 263-269, 2011.

4. Greene MF and Solomon CG: Gestational diabetes mellitus-time to treat. N Engl J Med 352: 2544-2546, 2005.

5. Elliott HR, Sharp GC, Relton CL and Lawlor DA: Epigenetics and gestational diabetes: A review of epigenetic epidemiology studies and their use to explore epigenetic mediation and improve prediction. Diabetologia 62: 2171-2178, 2019.

6. Fan Z, Cui H, Xu X, Lin Z, Zhang X, Kang L, Han B, Meng J, Yan Z, Yan X and Jiao S: MiR-125a suppresses tumor growth, invasion and metastasis in cervical cancer by targeting STAT3. Oncotarget 6: 25266-25280, 2015.

7. Xie F, Hosany S, Zhong S, Jiang Y, Zhang F, Lin L, Wang X, Gao S and Hu X: MicroRNA-193a inhibits breast cancer proliferation and metastasis by downregulating WT1. PLoS One 12: e0185565, 2017.

8. Wander PL, Boyko EJ, Hevner K, Parikh VJ, Tadesse MG, Sorensen TK, Williams MA and Enquobahrie DA: Circulating early- and mid-pregnancy microRNAs and risk of gestational diabetes. Diabetes Res Clin Pract 132: 1-9, 2017

9. Tagoma A, Alnek K, Kirss A, Uibo R and Haller-Kikkatalo K: MicroRNA profiling of second trimester maternal plasma shows upregulation of miR-195-5p in patients with gestational diabetes. Gene 672: 137-142, 2018

10. Floris I, Descamps B, Vardeu A, Mitić T, Posadino AM, Shantikumar S, Sala-Newby G, Capobianco G, Mangialardi G, Howard L, et al: Gestational diabetes mellitus impairs fetal endothelial cell functions through a mechanism involving microRNA-101 and histone methyltransferase enhancer of zester homolog-2. Arterioscler Thromb Vasc Biol 35: 664-674, 2015.

11. Lewis PW, Muller MM, Koletsky MS, Cordero F, Lin S, Banaszynski LA, Garcia BA, Muir TW, Becher OJ and Allis CD: Inhibition of PRC2 activity by a gain-of-function $\mathrm{H} 3$ mutation found in pediatric glioblastoma. Science 340: 857-861, 2013.

12. Xu CR, Li LC, Donahue G, Ying L, Zhang YW, Gadue P and Zaret KS: Dynamics of genomic H3K27me3 domains and role of EZH2 during pancreatic endocrine specification. EMBO J 33 2157-2170, 2014.

13. International Association of Diabetes and Pregnancy Study Groups Consensus Panel, Metzger BE, Gabbe SG, Persson B, Buchanan TA, Catalano PA, Damm P, Dyer AR, Leiva A, Hod M, et al: International association of diabetes and pregnancy study groups recommendations on the diagnosis and classification of hyperglycemia in pregnancy. Diabetes Care 33: 676-682, 2010.

14. Matsumoto T, Takaoka E, Ishida K, Nakayama N, Noguchi E, Kobayashi $\mathrm{T}$ and Kamata $\mathrm{K}$ : Abnormalities of endothelium-dependent responses in mesenteric arteries from Otsuka long-evans Tokushima fatty (OLETF) rats are improved by chronic treatment with thromboxane A2 synthase inhibitor. Atherosclerosis 205: 87-95, 2009.

15. Livak KJ and Schmittgen TD: Analysis of relative gene expression data using real-time quantitative PCR and the 2(-Delta Delta C(T)) method. Methods 25: 402-408, 2001

16. Zinman B, Genuth S and Nathan DM: The diabetes control and complications trial/epidemiology of diabetes interventions and complications study: 30 th anniversary presentations. Diabetes Care 37: 8, 2014.

17. Cefalu WT and Ratner RE: The diabetes control and complications trial/epidemiology of diabetes interventions and complications study at 30 years: The 'gift' that keeps on giving! Diabetes Care 37: 5-7, 2014.

18. Hales $\mathrm{CN}$ and Barker DJ: The thrifty phenotype hypothesis. Br Med Bull 60: 5-20, 2001.
19. Morwessel NJ: The genetic basis of diabetes mellitus. AACN Clin Issues 9: 539-554, 1998.

20. Pishavar E and Behravan J: miR-126 as a therapeutic agent for diabetes mellitus. Curr Pharm Des 23: 3309-3314, 2017.

21. Assmann TS, Duarte GCK, Brondani LA, de Freitas PHO, Martins EM, Canani LH and Crispim D: Polymorphisms in genes encoding miR-155 and miR-146a are associated with protection to type 1 diabetes mellitus. Acta Diabetol 54: 433-441, 2017.

22. Morais Junior GS, Souza VC, Machado-Silva W, Henriques AD, Melo Alves A, Barbosa Morais D, Nobrega OT, Brito CJ and Dos Santos Silva RJ: Acute strength training promotes responses in whole blood circulating levels of miR-146a among older adults with type 2 diabetes mellitus. Clin Interv Aging 12: 1443-1450, 2017.

23. Shan K, Liu C, Liu BH, Chen X, Dong R, Liu X, Zhang YY, Liu B, Zhang SJ, Wang JJ, et al: Circular noncoding RNA HIPK3 mediates retinal vascular dysfunction in diabetes mellitus. Circulation 136: 1629-1642, 2017.

24. Banerjee RR: Piecing together the puzzle of pancreatic islet adaptation in pregnancy. Ann N Y Acad Sci 1411: 120-139, 2018

25. Lane-Cordova AD, Gunderson EP, Carnethon MR, Catov JM, Reiner AP, Lewis CE, Dude AM, Greenland P and Jacobs DR Jr: Pre-pregnancy endothelial dysfunction and birth outcomes: The coronary artery risk development in young adults (CARDIA) study. Hypertens Res 41: 282-289, 2018

26. Magee TR, Ross MG, Wedekind L, Desai M, Kjos S and Belkacemi L: Gestational diabetes mellitus alters apoptotic and inflammatory gene expression of trophobasts from human term placenta. J Diabetes Complications 28: 448-459, 2014.

27. Li J, Ying H, Cai G, Guo Q and Chen L: Impaired proliferation of pancreatic beta cells, by reduced placental growth factor in pre-eclampsia, as a cause for gestational diabetes mellitus. Cell Prolif 48: 166-174, 2015

28. Tryggestad JB, Vishwanath A, Jiang S, Mallappa A, Teague AM, Takahashi Y, Thompson DM and Chernausek SD: Influence of gestational diabetes mellitus on human umbilical vein endothelial cell miRNA. Clin Sci (Lond) 130: 1955-1967, 2016.

29. Jin J, Wang C, Ouyang Y and Zhang D: Elevated miR-195-5p expression in deep vein thrombosis and mechanism of action in the regulation of vascular endothelial cell physiology. Exp Ther Med 18: 4617-4624, 2019

30. Xu J, Wang Z, Lu W, Jiang H, Lu J, Qiu J and Ye G: EZH2 promotes gastric cancer cells proliferation by repressing p21 expression. Pathol Res Pract 215: 152374, 2019.

31. Li Y, Li D, Zhao M, Huang S, Zhang Q, Lin H, Wang W, Li K, Li Z, Huang W, et al: Long noncoding RNA SNHG6 regulates p21 expression via activation of the JNK pathway and regulation of EZH2 in gastric cancer cells. Life Sci 208: 295-304, 2018.

32. Lian R, Ma H, Wu Z, Zhang G, Jiao L, Miao W, Jin Q, Li R, Chen P, Shi $\mathrm{H}$ and Yu W: EZH2 promotes cell proliferation by regulating the expression of RUNX3 in laryngeal carcinoma. Mol Cell Biochem 439: 35-43, 2018.

33. Yan JJ, Chang Y, Zhang YN, Lin JS, He XX and Huang HJ: miR-195 inhibits cell proliferation via targeting AEG-1 in hepatocellular carcinoma. Oncol Lett 13: 3118-3126, 2017.

34. Yin Y, Hong S, Yu S, Huang Y, Chen S, Liu Y, Zhang Q, Li Y and Xiao H: MiR-195 inhibits tumor growth and metastasis in papillary thyroid carcinoma cell lines by targeting CCND1 and FGF2. Int J Endocrinol 2017: 6180425, 2017.

35. Han Li C and Chen Y: Targeting EZH2 for cancer therapy: Progress and perspective. Curr Protein Pept Sci 16: 559-570, 2015.

36. Li Z, Hou P, Fan D, Dong M, Ma M, Li H, Yao R, Li Y, Wang G, Geng P, et al: The degradation of EZH2 mediated by lncRNA ANCR attenuated the invasion and metastasis of breast cancer. Cell Death Differ 24: 59-71, 2017.

37. Pepin ME, Bickerton HH, Bethea M, Hunter CS, Wende AR and Banerjee RR: Prolactin receptor signaling regulates a pregnancy-specific transcriptional program in mouse islets. Endocrinology 160: 1150-1163, 2019.

This work is licensed under a Creative Commons Attribution-NonCommercial-NoDerivatives 4.0 International (CC BY-NC-ND 4.0) License. 